\title{
PREVENCIÓN DE LA INFECCIÓN NEONATAL POR ESTREPTOCOCO DEL GRUPO B, ¿ES NECESARIA EN NUESTRO MEDIO?
}

\section{Prevention of perinatal group b streptococcal infection, is it necessary in our country?}

\author{
Hernán Cortés, M.D.* \\ Recibido: abril 6/05 - Revisado: junio 14/05 - Aceptado: agosto 12/05
}

\section{RESUMEN}

El Estreptococo del grupo B (GBS) o Streptococcus agalactie, es un microrganismo saprofito habitual de los tractos genitourinario y gastrointestinal del ser humano. Las embarazadas colonizadas pueden transmitir de manera vertical esta bacteria a sus hijos, favoreciendo el desarrollo de la infección neonatal temprana. La prevalencia de colonización materna es variable, entre 5 y 30\% dependiendo del país, la raza, edad y técnica de recolección de la muestra. Se presenta colonización entre el 40 a 70\% de los bebes nacidos de madres portadoras y de éstos entre el 1 y $2 \%$ desarrollarán la enfermedad invasora (sepsis, meningitis y/o neumonía). En Estados Unidos, la infección por GBS es la principal causa de morbimortalidad neonatal. Afortunadamente la mayoría de las infecciones pueden ser prevenidas con el uso de antibióticos profilácticos durante el trabajo de parto. Sin embargo, en nuestro medio desconocemos el impacto de esta patología y no se han implementado las estrategias de prevención.

Corresponde pues a la universidad apropiarse de la investigación y desarrollo de estrategias que permitan implementar dicha profilaxis en nuestro país.

Palabras clave: Estreptococo grupo b, enfermedad, profilaxis, colonización.

* Ginecoobstetra, profesor de perinatología, Departamento de Ginecología y Obstetricia. Universidad de Antioquia.

Correo electrónico: hcortes@mixmail.com

\section{SUMMARY}

Group B Streptococcus or agalactie (GBS) is a habitual saprophytic microorganism of the genitourinary and gastrointestinal tracts of human beings. Vertical transmission from the mother to the fetus, as occurs, favors the development of early onset disease in the neonate. The prevalence of maternal colonization is variable (5-30\%) depending on the country, race, age and technique of sampling used for culture. Approximately 40-70\% of neonates from mothers who are positive for GBS are colonized and of those, 1 to $2 \%$ will develop invasive disease (sepsis, meningitis or pneumonia). Fortunately, most of these infections can be prevented by prophylactic antibiotics during labor and delivery.

However, in our environment, the impact of this pathology is unknown, and strategies for prevention have not been implemented. Research in our country is needed to address this issue and implement diagnostic and treatment strategies.

Key words: Streptococcus group B, disease, prophylaxis, colonization.

\section{METODOLOGÍA}

Se realizó una búsqueda sistemática en las bases de datos médicas OVID y Medline, con las palabras "Prevention of perinatal group B streptococcal disease", entre los años 1990 - 2005 y se escogieron los artículos de revisión, las guías de manejo y los estudios con un adecuado 
diseño metodológico; además se revisaron algunos textos guía y estudios realizados en nuestro país.

El objetivo fue establecer el impacto de esta infección en el país y determinar si es necesario o no implementar estrategias de prevención.

\section{INCIDENCIA}

Desde su identificación en los años 70s, el Estreptococo del grupo B (GBS), constituye la principal causa de infección y muerte neonatal en Estados Unidos. ${ }^{1}$ Actualmente la incidencia de esta infección en ese país es de $1,8 \times 1.000$ nacidos vivos (7.600 casos al año), que generan una mortalidad aproximada del 6\% (380 casos al año); ${ }^{2,3}$ sin embargo, en los recién nacidos prematuros, las cifras de mortalidad por esta causa alcanzan hasta un $16 \%{ }^{4}$ Es de anotar que la mortalidad en los $70 \mathrm{~s}$ era cercana al $50 \%$; gracias a los avances en el cuidado neonatal, ha logrado disminuirse. ${ }^{5}$

En los niños la infección por GBS puede ser de inicio temprano $(<7$ días) o de inicio tardío $(>$ 7 días) presentándose como neumonía, sepsis o meningitis; el 25\% de los casos ocurre en prematuros. ${ }^{4} \mathrm{La}$ enfermedad de inicio temprano representa el 80\% de los casos y entre el 15 - 30\% de éstos, tendrán secuelas a largo plazo.

En nuestro medio se cuenta con algunos trabajos sobre la prevalencia de la infección por GBS. En el Hospital Universitario San Vicente de Paúl en el 2002 Restrepo y cols. ${ }^{6}$ encontraron una prevalencia de colonización materna de 8,6\%. Sin embargo, su trabajo incluyó sólo mujeres con factores de riesgo $(n=81)$, de éstas, un bebe desarrolló sepsis de inicio temprano.

En 1999, Trujillo y cols. ${ }^{7}$ realizaron un trabajo en el Hospital General de Medellín con 78 pacientes de las cuales 13 estaban colonizadas (17\%); ningún bebé presentó la enfermedad. En Bogotá en 1999 González y cols. ${ }^{8}$ estudiaron 180 gestantes con factores de riesgo, y reportaron una prevalencia para GBS del 13\%.

Estudios previos en el Hospital General de Medellín en 1989 y 1990 habían revelado una prevalencia de $0-1,5 \%,{ }^{7,9}$ cifras bajas en comparación con los reportes de Restrepo ya citados. El incremento puede deberse a un aumento real en la incidencia o a la mejoría en las técnicas de recolección y cultivo.

Sin embargo, no contamos con estadísticas confiables sobre la incidencia de la infección perinatal, debido a que no se han implementado programas de vigilancia de esta patología. Por ejemplo, en el Hospital Universitario San Vicente de Paúl durante el 2003, entre 763 ingresos a la UCI neonatal, se presentó una mortalidad del 6\% (47 pacientes) de los cuales 50\% (24), fueron debidos a sepsis clínica, aunque no se cuenta con una diferenciación entre infecciones neonatales tempranas o tardías. En solo tres casos se aisló el germen causante.

Las estadísticas nacionales del DANE (www. dane.gov.co) para este mismo periodo sólo reportan a nivel nacional 18 muertes neonatales tempranas por sepsis, lo cual muestra un claro subregistro.

\section{COLONIZACIÓN}

Se presenta colonización asintomática entre el 5 -30\% de las embarazadas, dependiendo del país, grupo étnico, edad y técnicas de recolección. ${ }^{10}$ Entre el $1-2 \%$ de los niños nacidos de madres colonizadas desarrollan la enfermedad, de éstos, el $89 \%$ se presentan como sepsis y el $10 \%$ como meningitis. ${ }^{4,10}$

\section{Factores de riesgo para enfermedad perinatal}

Diversas condiciones aumentan la probabilidad de que un bebé desarrolle la infección de inicio temprano, la principal es la colonización materna que incrementa el riesgo más de 29 veces, en comparación con los bebés de madres con cultivo negativo. ${ }^{10,11}$

Otros factores importantes son nombrados en la tabla 1. Éstos se asocian a un aumento en el riesgo de más de 7 veces. ${ }^{11}$ 
Tabla 1. Otros factores importantes que aumentan la probabilidad de infección por GBS de inicio temprano

- Nacimiento prematuro $(<37$ semanas $)$

- Ruptura de membranas $>18$ horas

- Fiebre durante el parto $\left(>38^{\circ} \mathrm{C}\right)$

- Bacteriuria por GBS durante el embarazo

- Antecedentes de bebé con infección por GBS

También se asocian con el desarrollo de la enfermedad, aunque en menor grado, la edad $<20$ años, raza negra, origen hispano, bajos niveles de anticuerpos contra el antígeno capsular del GBS y la colonización masiva. ${ }^{2,11}$ No se conocen con certeza los factores de riesgo para desarrollar la enfermedad de inicio tardío; muchas de éstas corresponden a infección nosocomial o adquirida en la comunidad. ${ }^{2,10}$

\section{PREVENCIÓN}

Debido a la severidad y frecuencia de la infección por GBS en recién nacidos, se han buscado métodos para prevenir la transmisión de la madre al bebé, principalmente mediante el uso de antibióticos. Estas estrategias de prevención se pueden dividir en anteparto, durante el parto, prevención inmunológica y prevención neonatal.

\section{Tabla 2. Sistema usado para calificar la evidencia que determina el nivel de recomendación.}

\begin{tabular}{|c|c|c|}
\hline CATEGORIA & DEFINICIÓN & RECOMENDACIÓN \\
\hline & Fuerza de la recomendación & \\
\hline A & $\begin{array}{l}\text { Hay buena evidencia que la medida es eficaz y } \\
\text { beneficiosa }\end{array}$ & $\begin{array}{l}\text { Fuertemente } \\
\text { recomendado }\end{array}$ \\
\hline $\mathrm{B}$ & $\begin{array}{l}\text { Evidencia buena o moderada de la eficacia, pero } \\
\text { los beneficios clínicos son limitados }\end{array}$ & $\begin{array}{l}\text { Generalmente } \\
\text { recomendado }\end{array}$ \\
\hline $\mathrm{C}$ & $\begin{array}{l}\text { Evidencia insuficiente de su eficacia o los beneficios } \\
\text { no superan los posibles riesgos }\end{array}$ & Opcional \\
\hline $\mathrm{D}$ & $\begin{array}{l}\text { Evidencia moderada de la ineficiencia de la medida, } \\
\text { o que los riesgos superan los beneficios }\end{array}$ & $\begin{array}{l}\text { Generalmente } \\
\text { no recomendado }\end{array}$ \\
\hline $\mathrm{E}$ & $\begin{array}{l}\text { Hay buena evidencia de su ineficiencia o de sus } \\
\text { riesgos. }\end{array}$ & No recomendado \\
\hline $\begin{array}{l}\text { Calidad de la evidencia que so- } \\
\text { porta la recomendación }\end{array}$ & & \\
\hline
\end{tabular}

Evidencia proveniente de al menos un estudio aleatorio y controlado bien realizado, o de un estudio experimental que ha sido validado por un investigador independiente

Evidencia de un estudio no aleatorizado, pero bien diseñado, o un estudio de casos y controles (preferiblemente de mas de un lugar), estudios no controlados con resultados altamente significativos o evidencia de estudios experimentales

Evidencia basada en opiniones de expertos, III soportada en su experiencia clínica, estudios descriptivos o reportes de comites de expertos.

Adaptado de: CDC , 1999 USPHS/IDSA guidelines for the prevention of opportunistic infections in persons infected with Human immunodeficiency virus. MMWR1999;48(rr10): 1-66. 


\section{Prevención anteparto}

El tratamiento antibiótico en las mujeres colonizadas no es efectivo, por lo que no se recomienda. ${ }^{4,10}$

\section{Prevención durante el parto}

A partir de los años 80s se demostró que la administración de antibióticos profilácticos durante el trabajo de parto en mujeres colonizadas por el GBS o con factores de riesgo, disminuía la incidencia de enfermedad en el recién nacido; ${ }^{12}$ sin embargo, la profilaxis intraparto no fue adoptada de manera universal hasta 1996, cuando la Academia Estadounidense de Pediatría (APA), el Colegio Estadounidense de Obstetras y Ginecólogos (ACOG) y el Centros para el Control de las Enfermedades (CDC), realizaron unas guías de manejo que se basaban en dos alternativas:

1. Toma de cultivo para GBS en las mujeres embarazadas entre la semana 35 a 37, brindando profilaxis a las mujeres positivas.

2. Con base en factores de riesgo durante el parto, a saber: trabajo de parto prematuro, ruptura de membranas $>18$ horas, fiebre durante el parto, entre otras.

Se recomendó el uso de la bencilpenicilina o ampicilina. $^{2}$

Con esta estrategia se redujo la incidencia de infección neonatal en aproximadamente 70\%, pasando de 2 - 3 casos/ 1.000 nacidos vivos a 0,5 casos/1.000 nacidos vivos. Además, la incidencia

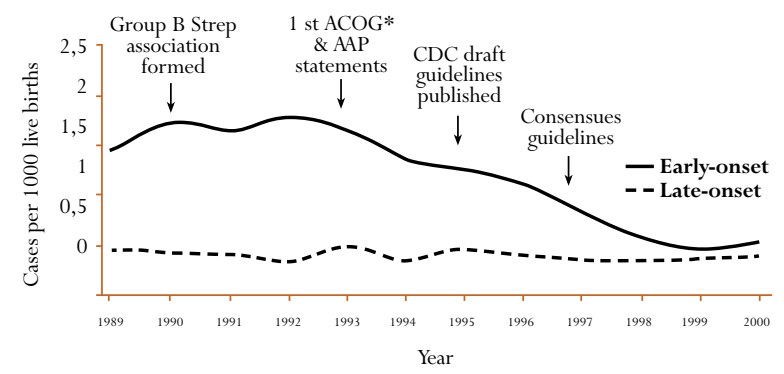

Gráfica 1. Incidencia de enfermedad temprana y tardía por Estreptococo invasivo del grupo B.

Fuente: Schrag S, Gorwitz R, Fultz-Butts K, Schuchat A. Prevention of perinatal group $B$ streptococcal disease. Revised guidelines from CDC. MMWR. 2002;5 1:1-22 Adaptado de Early onset group B streptococcal disease United States 19981999 MMWR 2000;49:793796 y Schrag SJ, Zywicki S, Farley MM, Reingold AL, Harrison LH, Lefkowitz LB, et al. Group $B$ streptococcal disease in the era of intrapartum antibiotic prophylaxis. $N$ Engl J Med 2000;342:15-20 de enfermedad invasora en las embarazadas (endometritis y amnionitis) disminuyó en 21\% entre 1993 y 1998; cabe anotar que la incidencia de la enfermedad tardía no ha variado. ${ }^{3}$ (gráfica 1).

Sin embargo, a pesar de la dramática disminución en la incidencia por infección por GBS en los 90s, éste continuó en los años posteriores siendo la principal causa de morbimortalidad neonatal en Estados Unidos, ${ }^{13}$ por lo que continuó el debate sobre cuál estrategia sería la más efectiva para identificar a las candidatas a profilaxis intraparto.

En el 2002, Schragy cols. ${ }^{14}$ realizaron un estudio con una población de más de 600.000 nacidos vivos, comparando la efectividad de las dos estrategias, encontrando que la prevención por medio de cultivo era un 50\% más efectiva que la estrategia basada en factores de riesgo; el beneficio de la detección sistémica se debe a dos motivos:

El primero es que permite la identificación de mujeres colonizadas sin factores de riesgo; en la cohorte de mujeres a quienes se les aplico la prueba de detección, el 18\% de las colonizadas no tuvieron factores de riesgo durante el parto.

El segundo es que la administración de antibióticos era más probable entre las mujeres con cultivo positivo, en comparación con las mujeres en el grupo con factores de riesgo.

Debido a esto, la CDC cambió las guías, ${ }^{10}$ tomando en consideración la nueva evidencia disponible. Las recomendaciones se enumeran a continuación, y el nivel de evidencia considerado se explica en la tabla 2.

- Se debe realizar tamizaje a toda mujer embarazada entre las semanas 35 y 37 para colonización rectal y vaginal. A las que se identifique como portadoras, se deberá brindar profilaxis durante el parto (AII).

- En mujeres en quienes se aísle GBS en cualquier concentración en orina, se deberá administrar profilaxis y no requieren prueba de detección (BII).

- Toda mujer con antecedente de un bebé al que se le diagnosticó enfermedad invasiva por GBS, requiere profilaxis (BII). 


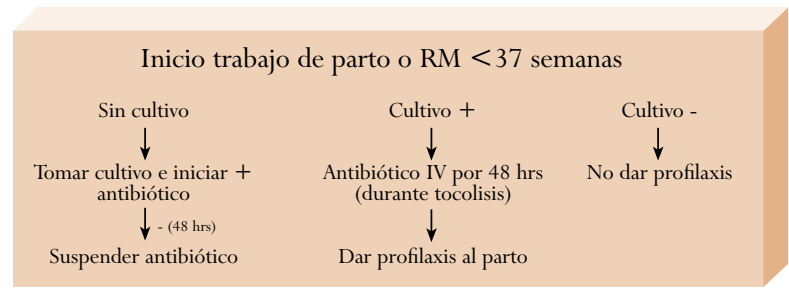

Gráfica 2. Recomendación para profilaxis en pacientes con amenaza de parto prematuro (APP) o ruptura prematura de membranas (RM).

Adaptado de Gibbs R, Schrag S, Schuchat A. Perinatal infections due to group B streptococci. Obstet Gynecol 2004; 104: 1062-76)

- Si no se conoce el resultado del cultivo al momento del parto se debe brindar profilaxis a todas las mujeres con uno de los siguientes factores de riesgo: embarazo menor de 37 semanas, ruptura de membranas mayor de 18 horas, o fiebre (temperatura $>38^{\circ} \mathrm{C}$ ); si cualquiera de los anteriores se presenta, pero si el cultivo es negativo, no se requiere profilaxis (AII).

- En las mujeres con trabajo de parto prematuro $(<37$ semanas) o ruptura prematura de membranas, se debe evaluar la necesidad de brindar profilaxis; se presenta una guía de manejo (gráfica 2).

- Realizar técnicas de cultivo y recolección adecuada para maximizar la posibilidad de detectar el GBS. Se deben tomar muestras de vagina y recto, que se transportarán y cultivarán en los medios adecuados (AII).

- No se debe tratar la colonización por GBS, pues no es una estrategia efectiva y sí puede tener efectos adversos (DI).
- Las mujeres a quienes se les realice cesárea electiva, no requieren profilaxis (CII).

- Para la profilaxis se recomienda el siguiente régimen: bencilpenicilina (penicilina G) 5 millones de unidades i.v. de dosis inicial y continuar 2,5 millones cada 4 horas hasta el parto (AII), un régimen alternativo es la ampicilina $2 \mathrm{~g}$ i.v. inicialmente y luego $1 \mathrm{~g}$ cada 4 horas hasta el parto. Se prefiere la penicilina por su espectro menos amplio (AII).

- Para las mujeres alérgicas a la penicilina, se recomienda cefazolina ( $2 \mathrm{~g}$ i.v. inicial y $1 \mathrm{~g}$ cada 8 horas), eritromicina (500 mg i.v. cada 6 horas) o clindamicina (900 mg i.v. cada 8 horas); si el GBS es resistente se debe usar vancomicina $1 \mathrm{~g}$ i.v. cada 12 horas.

- No se recomienda el uso rutinario de antibióticos en los hijos de madres que recibieron la profilaxis (CIII).

- Las agencias de salud deben promover y vigilar la aplicación de estas normas, para reducir la incidencia de enfermedad neonatal temprana por GBS.

El régimen recomendado por el CDC para la prevención de la infección neonatal temprana por GBS se presenta en la tabla 3.

\section{Prevención neonatal}

Aunque algunos autores reportan el uso de antibióticos en los recién nacidos de madres portadoras de GBS, que no recibieron profilaxis, esta estrategia no ha demostrado ser efectiva y por lo tanto, no se recomienda. ${ }^{15}$

\section{Tabla 3. Régimen recomendado para la prevención de la infección neonatal temprana por GBS.}

\begin{tabular}{|c|l} 
Recomendado & $\begin{array}{l}\text { Bensilpenicilina, } 5 \text { millones de unidades i.v. dosis inicial. Continuar } \\
\text { con } 2,5 \text { millones unidades i.v. cada } 4 \text { horas hasta el parto. }\end{array}$ \\
\hline Alternativo & $\begin{array}{l}\text { Ampicilina, } 2 \mathrm{~g} \text { i.v. inicialmente, luego } 1 \mathrm{~g} \text { i.v. cada } 4 \text { horas } \\
\text { hasta el parto. }\end{array}$ \\
\hline Alergia a la penicilina & $\begin{array}{l}\text { Cefazolina, } 2 \mathrm{~g} \text { i.v. inicialmente, luego } 1 \mathrm{~g} \text { i.v. cada } 8 \text { horas } \\
\text { hasta el parto. o clindamicina, } 900 \mathrm{mg} \text { i.v. cada } 8 \text { horas hasta el parto. } \\
\text { o eritromicina } 500 \mathrm{mg} \text { i.v. cada } 6 \text { horas, hasta el parto. }\end{array}$ \\
\hline GBS resistente en pacientes alérgicas & Vancomicina $1 \mathrm{~g}$ i.v. cada 12 horas \\
\hline
\end{tabular}

a GBS resistente a clindamicina o eritomicina, cuando se realice cultivo y pruebas de sensibilidad.

Adaptado de: CDC. Prevention of Perinatal Group B Streptococcal Disease. MMWR 2002; 51 (RR1 1); 1-22. 


\section{Prevención inmunológica}

El uso de los antibióticos ha disminuido la incidencia de la enfermedad neonatal de inicio temprano, pero no previene la infección de inicio tardío; tampoco tiene efecto en reducir la tasa de mortinatos por GBS, ni la enfermedad por GBS en adultos, por lo que el desarrollo de una vacuna sería lo ideal. Además se eliminaría la necesidad del tamizaje perinatal y los posibles riesgos y efectos adversos del antibiótico.

Actualmente se están desarrollando investigaciones de fase I y II, con resultados alentadores; sin embargo, aún hay limitantes. ${ }^{4,10,15}$

\section{EFECTOS ADVERSOS DE LA PROFILAXIS}

\section{Reacción alérgica y anafilaxia}

Cerca del 10\% de la población puede presentar reacciones alérgicas leves a la penicilina y en estos casos se recomienda el uso de cefazolina; la anafilaxia por penicilina es muy rara, se estima que sólo se presenta en 4 x 100.000 personas y en esta situación se recomienda la sustitución por otros antibióticos. ${ }^{10}$ Es importante que durante el control prenatal se aclare si la paciente es realmente alérgica.

\section{Resistencia del GBS a los antibióticos}

Hasta el momento no se ha demostrado resistencia del GBS a la penicilina o a la ampicilina, pero sí a la clindamicina y eritromicina, por lo que se recomienda realizar antibiograma a las pacientes alérgicas a la penicilina.

\section{Aumento en la incidencia de sepsis por otros microrganismos patógenos}

Actualmente no hay evidencia de aumento de sepsis temprana por otro microrganismo, principalmente por E. coli, que es la segunda causa de infección neonatal temprana. Sin embargo, algunos estudios han demostrado un incremento en la resistencia de estos microrganismos (E. coli y gram negativos) a la ampicilina, aunque limitado a los bebés de bajo peso $(401-1.500 \mathrm{~g}),{ }^{16}$ por lo que se insiste en el uso preferencial de la penicilina; también se ha observado esta resistencia en otras infecciones adquiridas en la comunidad, lo que sugiere que el aumento en la resistencia no se debe a la profilaxis del GBS. ${ }^{10,17}$

\section{TÉCNICAS DE CUILTIVO}

\section{Toma de la muestra}

- Mediante el uso de un aplicador se deben tomar dos muestras: una de la parte inferior de la vagina (orificio vaginal) y otra del recto, insertando el aplicador (que puede ser el mismo) a través del esfínter anal. La recolección puede ser realizada por la misma paciente siguiendo instrucciones adecuadas. No se debe usar espéculo, ni tomar muestra del cérvix.

- Los aplicadores deben ser transportados en un medio no nutritivo (i.e Amies o Stuart) y pueden ir juntos. Estos medios mantienen la viabilidad de la bacteria por 4 días.

- Se debe especificar que las muestras son para cultivar GBS, y si la paciente es alérgica a la penicilina, solicitar pruebas de sensibilidad a clindamicina y eritromicina, si se aísla el GBS.

\section{Procedimientos para el cultivo}

- Se debe esparcir la muestra del aplicador en un cultivo específico, suplementado con gentamicina y ácido nalidíxico, comercialmente conocidos como Todd-Hewitt o LIM.

- Cultivar por $18-24$ horas a $35^{\circ}-37^{\circ} \mathrm{C}$, con aire ambiental o $\mathrm{CO}_{2}$ al 5\%.

- Vigilar la aparición del microorganismo (zona de hemólisis, cocos gram + , catalasa negativos), si no aparecen a las 24 horas, reincubar e inspeccionar a las 48 horas.

- Se pueden usar algunas pruebas de detección de antígeno, para su identificación presuntiva.

- Realizar las pruebas de sensibilidad en caso de ser necesarias (alergia a penicilina). ${ }^{14}$ 


\section{PRUIEBAS DE DETECCIÓN RÁPIDA}

Actualmente existe un método de detección rápida aprobado por la FDA (Food and Drug Administration), el IDI-Strep B, Infectio Diagnostic Inc., Québec, Canadá, el cual se basa en la reacción en cadena de la polimerasa (PCR), para su uso en el momento del parto y que reemplazaría el cultivo entre las semanas 35-37, con una sensibilidad del 97\% y una especificidad del 100\%; sin embargo, no se ha comercializado en nuestro país, ni se ha implementado de manera universal en los países desarrollados. ${ }^{18}$

\section{MANEJO DEL RECIIÉN NACIDO}

Debido a la eficacia de la profilaxis durante el parto, no se recomienda tratamiento antibiótico rutinario a los recién nacidos de madres colonizadas. Es suficiente con observarlos por 24 horas, ya que en este período se presenta la enfermedad en el 90\% de los recién nacidos que la van a desarrollar, especialmente si se cumplen las siguientes condiciones:

- Recién nacido $>38$ semanas

- Profilaxis antibiótica $>4$ horas

Si no se cumplen estas condiciones, el recién nacido debe ser observado por al menos 48 horas. En caso de que el recién nacido presente signos de sepsis se debe iniciar el manejo antibiótico y los exámenes usuales. ${ }^{10}$

\section{CONCLUISIÓN}

En nuestro medio no es clara la incidencia de infección neonatal por estreptococo del grupo B, ni el peso que tiene sobre la morbimortalidad neonatal; lo que sí se conoce es que la prevalencia de colonización por GBS en las mujeres embarazadas oscila entre 9 y 17\%, como lo han demostrado algunos trabajos locales. Esta cifra se asemeja a la prevalencia en Estados Unidos, por lo que pudiéramos pensar que la tasa de transmisión y enfermedad en los bebés también deben ser importantes en nuestro país. Infortunadamente no se cuenta con estadísticas confiables que respalden tal afirmación, debido a que no se han implementado programas de vigilancia de esta infección.

La ausencia del medio de cultivo específico para GBS en Medellín constituye otro problema importante, ya que al usar los medios disponibles se obtienen resultados falsos negativos en el diagnóstico, en cifras incluso superiores al 50\%.

Finalmente, existe un desconocimiento general de las medidas de prevención y su impacto por parte de los médicos que asisten a mujeres embarazadas, debido a la falsa creencia que esta enfermedad no se presenta en nuestro medio y que es un problema limitado a Estados Unidos. Como ya se explicó, el problema probablemente reside en el diagnóstico y el subregistro.

Debido a esto, la Universidad de Antioquia está implementando en el servicio de Obstetricia del Hospital Universitario San Vicente de Paúl, la prevención basada en el cultivo rutinario entre las semanas 35 - 37 de gestación, con el fin de evaluar el impacto de esta medida sobre la morbimortalidad neonatal.

Se dará profilaxis según el resultado del cultivo, medida que ha demostrado tener una efectividad mayor al 50\%, en comparación con la profilaxis basada en factores de riesgo (debido a que el $20 \%$ de las mujeres colonizadas no presentarán factores de riesgo y no recibirán profilaxis durante el parto; además, al conocer el resultado del cultivo se logra una mayor adherencia a las guías). Así se evita el uso del antibiótico en aquellas pacientes con factores de riesgo pero sin colonización, disminuyendo la probabilidad de resistencia a los antibióticos y la emergencia de otros gérmenes como causales de esta entidad. Se pretende además extender estos procedimientos a todas las instituciones locales y del país.

Por último, es claro que la solución definitiva no reside en el uso de los antibióticos sino en el desarrollo de una vacuna, la cual, como se mencionó anteriormente, está todavía en proceso de investigación. 


\section{REFERENCIAS}

1. Franciosi RA, Knostman JD, Zimmerman RA. Group B streptococcal neonatal and infant infections. J Pediatr 1973;82:707-18.

2. CDC.prevention of Perinatal group B streptococcal disease: a public health perspective. MMWR Recomm Rep 1996;45:1-24.

3. Schrag SJ, Zywicki S, Farley MM, et al. Group B streptococcal disease in the era of intrapartum antibiotic prophylaxis. N Engl J Med 2000;342:15-20.

4. Gibbs R, Sweet R. Maternal and fetal infectious disorders. En: Creasy R, Resnik R. Maternal-Fetal Medicine. $4^{\mathrm{a}}$ Ed. Saunders Company. p. 674-77.

5. Gladstone IM, Ehrenkranz RA, Edberg SC. A ten year review of neonatal sepsis and comparison with the previous fifty year experience. Pediatr Infect Dis J 1990;9:819-25.

6. Restrepo A, Serna L, Vanegas C, et al. Prevalencia de Streptococcus agalactiae en gestantes con factores de riesgo y sus recién nacidos. Hospital Universitario San Vicente de Paúl. 2002. Infectio 2003;7:147-52.

7. Trujillo M, Ospina B, Fama M. Reevaluación del estado de colonización por streptococcus del grupo B en madres e hijos al momento del parto. Rev Ces Medicina 1999;13:44.

8. Gonzalez CP, Gonzalez JE. Prevalencia de Estreptococo beta hemolítico en mujeres embarazadas de alto riesgo en el Hospital Simón Bolívar de Bogotá. UCIN 2001;2:7-15.

9. Manotas RJ, Baquero D. Frecuencia de aislamiento de Streptococcus agalactiae en un grupo de embarazadas y sus productos. IATREIA 1989;2:111-13.
10. Schrag S, Gorwitz R, Fultz-Butts K, Schuchat A. Prevention of Perinatal Group B Streptococcal Disease Revised Guidelines from CDC. MMWR Recomm Rep 2002;16;51:1-22.

11. Schuchat A, Deaver RK, Plikaytis BD, et al. Multistate case-control study of maternal risk factors for neonatal group B streptococcal disease. Pediatr Infect Dis J 1994;13:623-9.

12. Boyer KM, Gotoff SP. Prevention of early-onset neonatal group B streptococcal disease with selective intrapartum chemoprophylaxis. N Engl J Med 1986;314:1665-9.

13. Zangwill KM, Schuchat A, Wenger JD. Group B streptococcal disease in the United States, 1990. MMWR CDC Surveill Summ 1992;41:25-32.

14.Schrag SJ, Zell RE, Lynfield R, Roome A, Arnold $\mathrm{KE}$, Craig AS, et al. A population based comparison of strategies to prevent early-onset group B streptococcal disease in neonates. $\mathrm{N}$ Engl J Med 2002;347:233-9.

15. Gibbs R, Schrag S, Schuchat A. Perinatal infections due to group B streptococci. Obstet Gynecol 2004; 104: 1062-76.

16. Stoll BJ, Hansen N, Fanaroff A, Wright LL, Carlo WA, Ehrenkranz EA, et al. Changes in pathogens causing early-onset sepsis in very low birth weight infants. N Engl J Med 2002; 347:240-7.

17. Gupta K, Scholes D, Stamm WE. Increasing prevalence of antimicrobial resistance among uropathogens causing acute uncomplicated cystitis in women. JAMA 1999;282:325-6.

18. Bergeron MG, Ke D, Menard C, et al. Rapid detection of group B streptococci in pregnant women at delivery. N Engl J Med 2000;343:175-9. 